\title{
Transversus Abdominis Muscle
}

National Cancer Institute

\section{Source}

National Cancer Institute. Transversus Abdominis Muscle. NCI Thesaurus. Code C53179.

An anterolateral muscle of the abdomen which attaches to the thoracolumbar fascia, iliac crest, inguinal lig ament, and the costal cartilages of the lower ribs, and which continues anteriorly as the transverse abdominis aponeurosis, which inserts into the linea alba, the pubic crest, and the pectineal line. 